\title{
Robust stability and performance analysis for multiple model adaptive controllers
}

\author{
Dominic Buchstaller and Mark French
}

\begin{abstract}
For an Estimation Based Multiple Model Switched Adaptive Control (EMMSAC) algorithm controlling a MIMO minimal LTI plant, $l_{p}, 1 \leq p \leq \infty$ bounds on the gain from the input and output disturbances to the internal signals are obtained which are invariant to the number of models in the plant model set. For a compact uncertainty set it is shown that a realisable EMMSAC algorithm achieves robust stability for any plant within the uncertainty set.
\end{abstract}

\section{INTRODUCTION}

The feature that distinguishes the EMMSAC algorithm [6], [13], [7], [3], [4] from alternative approaches to multiple model adaptive control in the style of [11], [10] is that the performance of candidate plant models is evaluated by estimating the size of the minimal disturbance signal which is compatible with the observed closed loop signals and the plant model instead of evaluating the output error of an observer.

Basing controller selection on such an disturbance estimation process allowed the construction of $l_{2}$ gain bounds on the gain from the input and output disturbances to the internal signals for two plant models in [6]; $l_{p}, 1 \leq p \leq \infty$ gain bounds for the class of dead beat stabilisable plants in [7]; gain function bounds for dead beat stabilisable plants which are invariant to the uncertainty of the system (nonconservative) in [3] and gain (function) bounds for the class of general MIMO minimal LTI systems in [4]. Note that in combination with [8], such bounds lead to a robust stability certificate, guaranteeing robustness for unstructured uncertainties described by the gap metric.

Whilst the gain bounds in [7], [3], [4] are applicable to multiple model schemes with arbitrarily large numbers of candidate plants, these upper bounds scale with the size of the candidate plant set. In some cases where the uncertainty set is large (for example containing a large number of non simultaneously stabilisable plants), this scaling is inevitable, and reflects the difficulty of the control problem. However in the case whereby a large number of plants modeled at high fidelity form the plant model set (as arises, for example, when modeling a parametric uncertainty by a discrete number of models partitioning the uncertainty set), the plant model set includes many plants which are close in the gap metric sense and hence it seems reasonable to expect that the true gain should scale rather better than the bounds in [7], [3], [4].

D. Buchstaller is with the School of Electrical and Electronic Engineering, Imperial College London, SW7 2AZ, UK, d.buchstaller@imperial.ac.uk

M. French is with the School of Electronics and Computer Science, University of Southampton, SO17 1BJ, UK, mcf decs. soton. ac.uk
The purpose of this paper is to show that this is indeed the case. We will also briefly discuss how such an analysis leads naturally into the design of candidate plant sets, an issue which is widely acknowledged to be a key outstanding issue [5], [1], see [2] for a fuller discussion.

\section{DeFinitions}

\section{A. Norms and signals}

Let $\mathcal{S}$ denote the signal space $\mathcal{S}=\operatorname{map}\left(\mathbb{N}, \mathbb{R}^{h}\right), h \in \mathbb{N}$. Define the norms

$$
\|a\|_{r}:=\left(\sum_{0 \leq i<\infty}|a(i)|^{r}\right)^{1 / r},\|a\|_{\infty}:=\sup _{0 \leq i<\infty}|a(i)|
$$

for $a \in \mathcal{S}, 1 \leq r<\infty$. The truncation operator $\mathscr{T}_{k}: \mathcal{S} \rightarrow \mathcal{S}$,

$$
\left(\mathscr{T}_{k} a\right)(i)=\left\{\begin{array}{cc}
a(i), & 0 \leq i \leq k \\
0, & \text { otherwise }
\end{array}, k \in \mathbb{N}\right.
$$

and the restriction operator $\mathscr{R}_{i, k}: \mathcal{S} \rightarrow \mathbb{R}^{h(i+1)}, i, k \in \mathbb{N}$

$$
\mathscr{R}_{i, k} a:=(a(k-i), a(k-i+1), \ldots, a(k-1), a(k))
$$

are the tools by which we will argue about finite intervals of signals. An operator $O: \mathcal{S} \rightarrow \mathcal{S}$ is said to be causal if it satisfies

$$
\mathscr{T}_{k} O \mathscr{T}_{k} v=\mathscr{T}_{k} O v, \forall k \in \mathbb{N}, v \in \mathcal{S} .
$$

Let $\mathcal{V}=l_{r}, 1 \leq r \leq \infty$ and let $\mathcal{V}_{e} \supseteq \mathcal{V}$ denote the extended space of possibly unbounded signals:

$\mathcal{V}=l_{r}, 1 \leq r \leq \infty, \mathcal{V}_{e}:=\left\{v \in \mathcal{S} \mid \forall k \in \mathbb{N}: \mathscr{T}_{k} v \in \mathcal{V}\right\}$.

\section{B. Plant and controller}

Given the input and output dimension $m, o \in \mathbb{N}$ define the input and output signal spaces $\mathcal{U}=\mathcal{V}^{m}, \mathcal{Y}=\mathcal{V}^{o}$ where we let $\mathcal{W}=\mathcal{U} \times \mathcal{Y}, \mathcal{W}_{e}=\mathcal{U}_{e} \times \mathcal{Y}_{e}$. A plant $P: \mathcal{U}_{e} \rightarrow \mathcal{Y}_{e}$ is said to be in closed loop with a controller $C: \mathcal{Y}_{e} \rightarrow \mathcal{U}_{e}$ if they satisfy

$$
\begin{aligned}
& y_{1}=P u_{1}, u_{0}=u_{1}+u_{2}, y_{0}=y_{1}+y_{2}, \\
& u_{2}=C y_{2}
\end{aligned}
$$

as depicted in Figure 1. For notational convenience we often write $w_{0}=\left(u_{0}, y_{0}\right)^{\top} \in \mathcal{W}$ for input and output disturbances, $w_{1}=\left(u_{1}, y_{1}\right)^{\top} \in \mathcal{W}_{e}$ for plant in- and outputs and $w_{2}=$ $\left(u_{2}, y_{2}\right)^{\top} \in \mathcal{W}_{e}$ for observed signals or observation. By abuse of notation we let $w_{0}(-k)=w_{1}(-k)=w_{2}(-k)=$ $0, \forall k \in \mathbb{N} \backslash\{0\}$. 


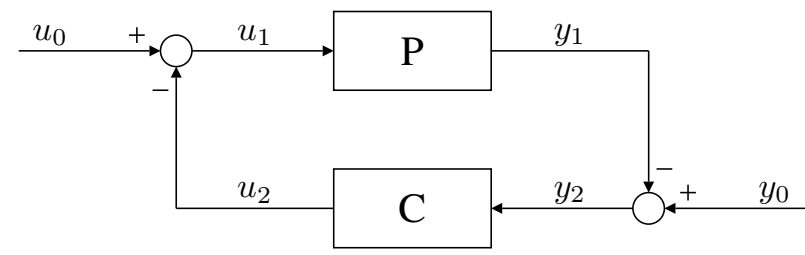

Fig. 1. The closed loop system $[P, C]$

Let $\mathcal{P}, \mathcal{C}$ be (plant, controller) parameter sets parametrising the class of all causal, MIMO minimal LTI systems. For all $p \in \mathcal{P}, c \in \mathcal{C}$ define the plant and controller operators

$$
\begin{aligned}
& P_{p}: \mathcal{U}_{e} \rightarrow \mathcal{Y}_{e}, P_{p}(-k)=0, k \in \mathbb{N} \\
& C_{c}: \mathcal{Y}_{e} \rightarrow \mathcal{U}_{e}, C_{c}(-k)=0, k \in \mathbb{N}
\end{aligned}
$$

and let

$$
\begin{aligned}
u_{2}^{c} & =C_{c} y_{2}^{c} \\
y_{1}^{p} & =P_{p} u_{1}^{p} \\
u_{0}^{p} & =u_{1}^{p}+u_{2} \\
y_{0}^{p} & =y_{1}^{p}+y_{2} .
\end{aligned}
$$

Define $\sigma(p), p \in \mathcal{P}$ to be the number of time steps the signal $w_{1}$ needs to be observed to uniquely determine the plant's initial condition. Define $\sigma(c), c \in \mathcal{C}$ with respect to the signal $w_{2}^{c}=\left(u_{2}^{c}, y_{2}^{c}\right)^{\top}$ accordingly.

\section{The closed loop operator}

Since we are interested in the gain of a system from the disturbance inputs $w_{0}$ to the internal signals $w_{2}$ we introduce the closed-loop operator

$$
\Pi_{C / / P}: \mathcal{W} \rightarrow \mathcal{W}_{e}: w_{0} \mapsto w_{2}
$$

where the gain is defined by

$$
\left\|\Pi_{C / / P}\right\|:=\sup _{w_{0} \in \mathcal{W} \backslash\{0\}} \frac{\left\|\Pi_{C / / P} w_{0}\right\|}{\left\|w_{0}\right\|} .
$$

We say that the closed loop $[P, C]$ is gain stable if $\left\|\Pi_{C / / P}\right\|<\infty$. Note that well posedness and boundedness of $w_{2}$ implies boundedness of $w_{1}$.

Theorem 2.1: Let $\mathcal{U}=\mathcal{Y}=l_{r}, 1 \leq r \leq \infty$. Let $P_{p_{1}}, P_{p_{2}} \in \operatorname{map}\left(\mathcal{U}_{e}, \mathcal{Y}_{e}\right), C \in \operatorname{map}\left(\mathcal{Y}_{e}, \mathcal{U}_{e}\right)$ and assume the closed loop $\left[P_{p_{i}}, C\right], i=\{1,2\}$ to be well-posed. Let the closed loop $\left[P_{p_{1}}, C\right]$ be gain stable. If

$$
\vec{\delta}\left(p_{1}, p_{2}\right)<\frac{1}{\left\|\Pi_{P_{p_{1}} / / C}\right\|}=b_{P_{p_{1}}, C}
$$

then the closed loop system $\left[P_{p_{2}}, C\right]$ is gain stable and

$$
\left\|\Pi_{P_{p_{2}} / / C}\right\| \leq\left\|\Pi_{P_{p_{1}} / / C}\right\| \frac{1+\vec{\delta}\left(p_{1}, p_{2}\right)}{1-\left\|\Pi_{P_{p_{1}} / / C}\right\| \vec{\delta}\left(p_{1}, p_{2}\right)}
$$

where $\vec{\delta}$ denotes the directed gap.

Proof The proof can be found in [8].

\section{The disturbance estimator}

The following axiomatic treatment of the disturbance estimator in the form of five abstract assumptions has the virtue of detaching the stability and robustness analysis from the problem of constructing (effective) disturbance estimation algorithms in practice.

Define the estimation operator

$$
E: \mathcal{W}_{e} \rightarrow \operatorname{map}\left(\mathbb{N}, \operatorname{map}\left(\mathcal{P}, \operatorname{map}\left(\mathbb{N}, \mathbb{R}^{h}\right)\right)\right)
$$

for $k \in \mathbb{N}, p \in \mathcal{P}$ by

$$
w_{2} \mapsto\left[k \mapsto\left(p \mapsto d_{p}[k]\right)\right]
$$

where $d_{p}[k]$ represents a time series of disturbance estimates up to time $k$ corresponding to a plant $p \in \mathcal{P}$. Explicitly let

$$
d_{p}[k]: \mathbb{N} \rightarrow \operatorname{map}\left(\mathbb{N}, \mathbb{R}^{h}\right)
$$

be defined by

$$
d_{p}[k]=\left(d_{p}[k](0), d_{p}[k](1), \ldots, d_{p}[k](k), 0, \cdots\right)
$$

where $h \in \mathbb{N}$ depends on $p \in \mathcal{P}$. Since we are interested in the 'size' of disturbance estimates, define the norm operator

$$
\begin{aligned}
N: \operatorname{map}\left(\mathbb{N}, \operatorname{map}\left(\mathcal{P}, \operatorname{map}\left(\mathbb{N}, \mathbb{R}^{h}\right)\right)\right) & \\
& \rightarrow \operatorname{map}\left(\mathbb{N}, \operatorname{map}\left(\mathcal{P}, \mathbb{R}^{+}\right)\right)
\end{aligned}
$$

by

$$
\left[k \mapsto\left(p \mapsto d_{p}[k]\right)\right] \mapsto\left[k \mapsto\left(p \mapsto\left\|d_{p}[k]\right\|\right)\right] .
$$

We denote the object $N E: \mathcal{W}_{e} \rightarrow \operatorname{map}\left(\mathbb{N}, \operatorname{map}\left(\mathcal{P}, \mathbb{R}^{+}\right)\right)$ the disturbance estimator. Let

$$
W_{p}(i, k):=\left\{\begin{aligned}
& v \in \mathbb{R}^{m(i+1)} \times \mathbb{R}^{n(i+1)} \\
& \exists\left(u_{0}^{p}, y_{0}^{p}\right)^{\top} \in \mathcal{W}_{e} \text { s.t. } \\
& \mathscr{R}_{i, k} P_{p}\left(u_{0}^{p}-u_{2}\right)=\mathscr{R}_{i, k}\left(y_{0}^{p}-y_{2}\right) \\
& v=\left(\mathscr{R}_{i, k} u_{0}^{p}, \mathscr{R}_{i, k} y_{0}^{p}\right)
\end{aligned}\right\}
$$

denote the set of 'weakly' consistent disturbance signals at time $k$ of length $i$ to a plant $p \in \mathcal{P}$ and the observation $\left(u_{2}, y_{2}\right)^{\top}$, that are the disturbance signals that satisfy (2.7),(2.8) over an interval of length $i$. A vector $v \in \mathbb{R}^{m(i+1)} \times \mathbb{R}^{o(i+1)}$ is said to be weakly consistent if and only if $v \in W_{p}(i, k)$ for some $i, k \in \mathbb{N}, p \in \mathcal{P}$.

Let $p_{*}$ be the parameter corresponding to the "true" unknown plant $P:=P_{p_{*}} \in \mathcal{P}$. We now state the following five estimator assumptions:

Assumption 2.2: Let $\lambda \in \mathbb{R}$ be given.

1) (Causality): $E$ is causal.

2) (Minimality): There exists a $\mu>0$ such that for all $k \geq$ 0 , for $p \in \mathcal{P}$ and for all $\left(w_{0}, w_{1}, w_{2}\right) \in \mathcal{W} \times \mathcal{W}_{e} \times \mathcal{W}_{e}$ satisfying (2.1) for $P=P_{p}$

$$
N E\left(w_{2}\right)(k)(p)=\left\|E\left(w_{2}\right)(k)(p)\right\| \leq \mu\left\|w_{0}\right\| .
$$

3) (Weak consistency): Let $0 \leq j \leq \lambda$. For all $p \in \mathcal{P}$ there exist maps

$$
\Phi_{j}: \operatorname{map}\left(\mathbb{N}, \mathbb{R}^{h}\right) \rightarrow \mathbb{R}^{m(j+1)} \times \mathbb{R}^{o(j+1)},
$$


such that for all $\left(w_{0}, w_{1}, w_{2}\right) \in \mathcal{W} \times \mathcal{W}_{e} \times \mathcal{W}_{e}$ satisfying (2.1) for $P=P_{p}$ and for all $k \in \mathbb{N}$,

$$
\Phi_{j} E\left(w_{2}\right)(k)(p) \in W_{p}(j, k)
$$

and

$$
\left\|\Phi_{j} E\left(w_{2}\right)(k)(p)\right\| \leq\left\|\mathscr{R}_{j, k} E\left(w_{2}\right)(k)(p)\right\| .
$$

4) (Monotonicity): For all $p \in \mathcal{P}$, for all $k, l \in \mathbb{N}$ with $0 \leq k \leq l$ and for all $\left(w_{0}, w_{1}, w_{2}\right) \in \mathcal{W} \times \mathcal{W}_{e} \times \mathcal{W}_{e}$ satisfying (2.1) for $P=P_{p}$ there holds

$$
\left\|E\left(w_{2}\right)(k)(p)\right\| \leq\left\|\mathscr{T}_{k} E\left(w_{2}\right)(l)(p)\right\| .
$$

5) (Continuity): There exists a $c: \mathbb{Z} \rightarrow \mathbb{R},\|c\|<\infty$ and a function $\chi: \mathcal{P} \times \mathcal{P} \rightarrow \mathbb{R}^{+} \cup\{\infty\}, \chi(p, p)=0$ such that for all $p_{1}, p_{2} \in \mathcal{P}$ and $w_{2} \in \mathcal{W}_{e}$ there holds:

$$
\begin{aligned}
& \left\|\Phi_{j} E\left(w_{2}\right)(k)\left(p_{1}\right)-\Phi_{j} E\left(w_{2}\right)(k)\left(p_{2}\right)\right\| \\
& \quad \leq \chi\left(p_{1}, p_{2}\right)\left\|\Upsilon_{k} w_{2}\right\|, \quad 0 \leq j \leq \lambda, k \in \mathbb{N}
\end{aligned}
$$

where $\Phi_{j}$ is as in Assumption 3 and

$$
\left(\Upsilon_{k} w_{2}\right)(i)=\left\{\begin{array}{cc}
c(k-i) w_{2}(i) & \text { if } \\
0 & \text { else }
\end{array} \quad i \leq k .\right.
$$

Note that Assumptions 1-4 are as in [4]. The intuitive meaning of the additional Assumption 5 is that if two plants are close to each other, their disturbance estimates are required to be 'close' and the effect of $w_{2}$ on the disturbance estimate needs to diminish over time.

An important class of estimator (called estimator B in [7], [3], [4]) is then given as follows. Let $k, \lambda, i \in \mathbb{N}, 0 \leq i \leq k$. To a plant model $P_{p}, p \in \mathcal{P}$ let estimator B with $h=$ $(m+o)(\lambda+1)$ in equation (2.9) be given by:

$$
\begin{aligned}
E_{B}\left(w_{2}\right)(k)(p) & =d_{p}^{B}[k] \in \operatorname{map}\left(\mathbb{N}, \mathbb{R}^{h}\right) \\
d_{p}^{B}[k](i) & =\underset{w_{0} \in W_{p}(\lambda, i)}{\operatorname{argmin}}\left\|w_{0}\right\|,
\end{aligned}
$$

if there exists a unique minimum, or any $d_{p}^{B}[k](i)$ satisfying

$$
\begin{aligned}
& d_{p}^{B}[k](i) \in\left\{\left.w_{0} \in \mathcal{W}\right|_{[i-\lambda, i]} \mid\right. \\
& \left\|w_{0}\right\|=\inf \left\{r \geq 0 \mid r=\left\|v_{0}\right\|, v_{0} \in W_{p}(\lambda, i)\right\}
\end{aligned}
$$

if the minimum is not unique, where $W_{p}(\lambda, i)$ is the set of all disturbance signals consistent with the observation $w_{2}$ and the plant $P_{p}$ over the interval $[i-\lambda, i]$.

Lemma 2.3: Estimator B meets Assumptions 2.2.

Proof: 1-4 can be found in [4]. 5. Let $p_{1}, p_{2} \in \mathcal{P}, k \in$ $\mathbb{N}, w_{2} \in \mathcal{W}_{2}$. Then

$$
\begin{aligned}
\left|\left\|\Phi_{j} d_{p_{1}}^{B}[k]\right\|-\left\|\Phi_{j} d_{p_{2}}^{B}[k]\right\|\right| & \leq\|\| \Phi_{j} d_{p_{1}}^{B}[k]-\Phi_{j} d_{p_{2}}^{B}[k] \| \mid \\
& \leq \chi\left(p_{1}, p_{2}\right)\left\|\Upsilon_{k} w_{2}\right\|
\end{aligned}
$$

where

$$
\chi\left(p_{1}, p_{2}\right)=\left\{\begin{array}{cl}
0 & \text { if } p_{1}=p_{2} \\
\infty & \text { if not }
\end{array}\right.
$$

for some $\Upsilon$ with $\|c\|<\infty$ as required.

We will later require $\chi$ to be continuous.
Conjecture 2.4: Let $1<r<\infty$. Suppose $\Omega \subset \mathcal{P}$ is compact. There exists $\chi$ that satisfies Assumption 2.2(5) and such that $\left.\chi\right|_{\Omega}$ continuous.

Continuity is expected to follow from the well-posedness of the underlying optimisation problem.

Note that the computational complexity of this estimator is independent of $k$ and reduces to standard optimisations with many possible implementations, e.g. via the calculation of a suitable pseudo inverse in $l_{2}$ or via linear programming in $l_{1}$ or $l_{\infty}$.

\section{E. Finite horizon behaviour of the closed loop $\left[P_{p}, C_{c}\right]$}

In order to assign a stabilising controller to each plant model we employ a so called controller design procedure

$$
K: \mathcal{P} \rightarrow \mathcal{C}
$$

Analogously to the estimator assumptions we now state two abstract (controller) assumption on the atomic closed loop systems $\left[P_{p}, C_{c}\right]$ and $\left[P_{p}, C_{K(p)}\right]$ :

Assumption 2.5: There exist functions

$$
\alpha, \beta: \mathcal{P} \times \mathcal{C} \times \mathbb{R} \times \mathbb{R} \rightarrow \mathbb{R}
$$

such that the following holds:

1) (Linear growth of $\left[P_{p}, C_{c}\right]$ ): Let $p \in \mathcal{P}, c \in$ $\mathcal{C}$ and the closed loop $\left[P_{p}, C_{c}\right]$ be well-posed. Let $l_{1}, l_{1}, l_{2}, l_{3}, l_{4} \in \mathbb{N}, l_{1}<l_{2} \leq l_{3}<l_{4}$ and $I_{1}=\left[l_{1}, l_{2}\right), I_{2}=\left[l_{2}, l_{3}\right), I_{3}=\left[l_{3}, l_{4}\right)$. Suppose $w_{2}, w_{2}^{c}, w_{1}^{p} \in \mathcal{W}_{e}, w_{0}^{p} \in \mathcal{W}$ satisfy equations (2.5)(2.8) on $I_{1} \cup I_{2} \cup I_{3}$.

Suppose $\left.w_{2}^{c}\right|_{I_{1}} \in\left\{0,\left.w_{2}\right|_{I_{1}}\right\},\left.\quad w_{2}^{c}\right|_{I_{2} \cup I_{3}}=\left.w_{2}\right|_{I_{2} \cup I_{3}}$ where

$$
\left|I_{1}\right|=l_{2}-l_{1} \geq \max \{\sigma(p), \sigma(c)\}
$$

Then:

$$
\begin{aligned}
\left\|\left.w_{2}\right|_{I_{3}}\right\| \leq \alpha\left(p, c,\left|I_{2}\right|,\left|I_{3}\right|\right)\left\|\left.w_{2}\right|_{I_{1}}\right\| \\
+\beta\left(p, c,\left|I_{2}\right|,\left|I_{3}\right|\right)\left\|\left.w_{0}^{p}\right|_{I_{1} \cup I_{2} \cup I_{3}}\right\| .
\end{aligned}
$$

2) (Stability of $\left.\left[P_{p}, C_{K(p)}\right]\right)$ : Let $p \in \mathcal{P}$ and $x \in \mathbb{N}$. Then

$$
\alpha(p, K(p), a, x) \rightarrow 0 \text { as } a \rightarrow \infty
$$

and $\alpha$ is monotonic in $a$.

We note that the above assumptions are in fact standard properties for minimal LTI systems. Plants and controllers satisfy Assumption 2.5(1), and stabilising LTI control design procedures $K$ satisfy Assumption 2.5(2).

\section{F. The switching algorithm}

The set of candidate plants model set that are available for consideration at time step $k \in \mathbb{N}$ is specified by $G(k)$. The time varying nature of the operator $G$ has been shown in [3], [4] to be the key to establish a non-conservative gain function bound for uncertainty sets which are unbounded. In the main result in Section 4, we specialise to the case of a constant $G$, however, we have retained the general time varying case in what follows next for consistency with the previous results, and for future development of these results. 
Let $\mathcal{P}^{*}$ be the powerset of $\mathcal{P}$. Let $\emptyset \neq \mathcal{P}_{i} \in \mathcal{P}^{*}, i \in \mathbb{N}$.

Definition 2.6: A map $G: \mathcal{W}_{e} \mapsto \operatorname{map}\left(\mathbb{N}, \mathcal{P}^{*}\right)$ is said to be a plant generating operator if it is causal and satisfies

$$
G(0)=\mathcal{P}_{1}, G(k)=\mathcal{P}_{i(k)}, k \in \mathbb{N}
$$

for some $i: \mathbb{N} \rightarrow \mathbb{N}$ with $i(0)=1$. $G$ is said to be feasible if $\mathcal{P}_{i}$ is a finite set for all $i \in \mathbb{N}$ and constant if $\mathcal{P}_{i}=$ $\mathcal{P}_{j}, \forall i, j \in \mathbb{N}$.

To improve readability we write $G(k):=G\left(w_{2}\right)(k), k \in$ $\mathbb{N}$, and if $G$ is constant then we also (by abuse of notation) let $G$ denote the set in question, i.e. we let $G=\mathcal{P}_{i}=\mathcal{P}_{j}$ for all $i, j \in \mathbb{N}$. Note that in any direct realisation of an EMMSAC algorithm, we will require that $\mathcal{P}_{i}$ is a finite set, but allowing more general plant sets to be considered is important in what follows.

We now would like the 'free' switching signal $q_{f}(k)$ to point to the plant model in $G(k)$ which corresponding disturbance estimate is minimal at time $k \in \mathbb{N}$. Hence let the minimisation operator

$$
M:\left(\operatorname{map}\left(\mathbb{N}, \operatorname{map}\left(\mathcal{P}, \mathbb{R}^{+}\right)\right), \operatorname{map}\left(\mathbb{N}, \mathcal{P}^{*}\right)\right) \rightarrow \operatorname{map}\left(\mathbb{N}, \mathcal{P}^{*}\right)
$$

be given by

$$
\left[k \mapsto\left(p \mapsto r_{p}[k]\right),(k \mapsto G(k))\right] \mapsto\left[k \mapsto q_{f}(k)\right]
$$

where

$$
q_{f}(k)=\underset{p \in G(k)}{\operatorname{argmin}} r_{p}[k] .
$$

If there are multiple minimising residuals, an arbitrary ordering on $G(k)$ is imposed a priori, i.e. $G(k)=$ $\left\{p_{1}, p_{2}, \cdots, p_{n}\right\}$, and $\operatorname{argmin}_{p \in G(k)} r_{p}[k]$ is defined to return the parameter $p_{i} \in G(k)$ with the smallest index $i$ such that $r_{p_{i}}[k]$ is minimal. Since we will later utilise such a signal for controller selection and overly fast switching even between stabilising controllers can lead to instability, see [10], we introduce a suitable delay $\Delta$ :

Given a 'transition delay' function $\Delta: \mathcal{P} \rightarrow \mathbb{N}$ define the delay operator

$$
D: \operatorname{map}(\mathbb{N}, \mathcal{P}) \rightarrow \operatorname{map}(\mathbb{N}, \mathcal{P})
$$

by

$$
\left[k \mapsto q_{f}(k)\right] \mapsto[k \mapsto q(k)]
$$

where

$$
q(k)=\left\{\begin{array}{ccc}
q_{f}(k) & \text { if } & k-k_{s}(k) \geq \Delta\left(q\left(k_{s}(k)\right)\right) \\
q\left(k_{s}(k)\right) & \text { else }
\end{array}\right.
$$

and where $k_{s}: \mathbb{N} \rightarrow \mathbb{N}$ is given by:

$$
k_{s}(k)=\underset{0 \leq i \leq k}{\operatorname{argmax}} q(i) \neq q(i-1) .
$$

The purpose of the transition delay $D$ is to delay the free switching signal $q_{f}(k)$ for long enough to overcome the destabilising effect overly rapid switching. Let $x, y, c \in \mathbb{R}$, define

$$
\lfloor c\rfloor:=\max \{n \in \mathbb{Z} \mid n \leq c\} \text { and }\left(\begin{array}{l}
x \\
y
\end{array}\right):=\frac{x !}{y !(x-y) !}
$$

and let $J: \mathbb{N} \rightarrow \mathbb{N}$ be defined by

$$
J(\xi)=\xi\left(\begin{array}{c}
\xi \\
\lfloor\xi / 2\rfloor
\end{array}\right) .
$$

Let

$$
\sigma=\max \{\sigma(p), \sigma(K(p))\}, p \in \mathcal{P}
$$

and let $K: \mathcal{P} \rightarrow \mathcal{C}$ and the attenuation function $l: \mathcal{P} \rightarrow$ $[0,1)$ be given. Now choose the delay $\Delta$ such that

$$
J(r) \alpha^{r}(p, K(p), \Delta(p)-\sigma, \sigma) \leq l(p)<1, \forall p \in \mathcal{P}
$$

if $1 \leq r<\infty$ and

$$
\alpha(p, K(p), \Delta(p)-\sigma, \sigma) \leq l(p)<1, \forall p \in \mathcal{P}
$$

if $r=\infty$.

In practice one would choose a stabilising design procedure $K$ and some $l: \mathcal{P} \rightarrow[0,1)$ and then compute for all $p \in \mathcal{P}$ a corresponding $\Delta(p)$ such that inequality (2.20) for $l_{r}, 1 \leq r<\infty$ or inequality (2.21) for $l_{\infty}$ hold, hence we note that there always exists such a $\Delta$.

Finally define the switching operator

$$
\begin{aligned}
S: \mathcal{W}_{e} & \rightarrow \operatorname{map}(\mathbb{N}, \mathcal{P}): w_{2} \mapsto q \\
S & =\operatorname{DM}(N E, G) .
\end{aligned}
$$

and the switching controller

$$
C: \mathcal{Y}_{e} \rightarrow \mathcal{U}_{e}: y_{2} \mapsto u_{2}
$$

for all $k \in \mathbb{N}$ by

$$
u_{2}(k)=C_{K(q(k))}\left(y_{2}-\mathscr{T}_{k_{s}(k)-1} y_{2}\right)(k) .
$$

Equation (2.23) therefore ensures a zero initial condition for the atomic controller $C_{K(q(k))}$ when it is switched into closed loop at time $k_{s}(k), k \in \mathbb{N}$.

\section{STABILITY OF THE CLOSED LOOP SYSTEM}

Our objective is to establish $l_{r}, 1 \leq r \leq \infty$ bounds on the gain from the external disturbances $w_{0} \in \mathcal{W}$ to the internal signals $w_{2} \in \mathcal{W}_{e}$ for an arbitrary number of plants.

Let $U \subset \mathcal{P}$ specify an uncertainty set we seek to control. For example if we seek to control a plant

$$
P: y_{1}(k+1)=a y_{1}(k)+b u_{1}(k)
$$

where $b=1, a \in\left[-a_{\max }, a_{\max }\right]$ is an uncertain parameter and $a_{\max }<\infty$, then the results will apply with the continuum $U=\left[-a_{\max }, a_{\max }\right]$. On the other hand, if $a$ is known and $b \in\{-1,+1\}$ then the result can also be applied with $U$ taken to be the discrete set $U=\{-1,+1\}$. A naive application of Theorem 3.3 below with $G=U$ (or equivalently using the previous results [7], [3], [4]) yields a finite gain bounds for the latter case, but not the former. Furthermore, if $U$ is a continuum and $G=U$, then the resulting controller is based on an infinite number of plant estimators/controllers and is unrealisable. Hence we introduce a mechanism which allows us to establish gain 
bounds where $G$ is a finite sampled subset of $U$, for example in the above example by taking $G=R_{l}$ where

$$
R_{l}=\left[-a_{\max }, a_{\max }\right] \cap\left[\cdots,-\frac{2}{l},-\frac{1}{l}, 0, \frac{1}{l}, \frac{2}{l}, \cdots\right]
$$

for some suitable value of $l>0$. Our final result Theorem 4.3 gives a common gain bound for all values of $l>0$ above a critical threshold. This contrasts to the previous results [7], [3], [4] applied with the choice $G=R_{l}$ which give gain bounds which grow unboundedly with $l>0$ (note that this is equivalent to taking $G=U=R_{l}$ in Theorem 3.3 below).

Let $H \subset U \subset \mathcal{P}$. Let $\nu: \mathbb{N} \rightarrow \mathbb{R}^{+}$be given. Now define the ball

$$
\begin{aligned}
& B_{\chi}(p, \nu(p))=\{p\} \cup\left\{p_{1} \in \mathcal{P} \mid\right. \\
&\left.\chi\left(p, p_{1}\right)<\nu(p)\right\} \cap U, p \in \mathcal{P}
\end{aligned}
$$

to be the set of plants that reside within a neighbourhood of radius $\nu(p)$, as measured by $\chi$, around $p \in H$ in $U$. For an appropriate choice of $H$ and $\nu$, the union of the corresponding neighbourhoods in $U$ then leads to a cover for $U$ :

Definition 3.1: $(H, \nu)$ is said to be a cover for $U$ if:

$$
\left.U \subset R:=\cup_{p \in H} B_{\chi}(p, \nu(p))\right) .
$$

$(H, \nu)$ is said to be a finite cover if $H$ is a finite set.

The introduction of $(H, \nu)$ is the device by which we are able to express gain bounds in terms of the cover of the candidate plant set rather then the absolute size of the set $|G|$.

Definition 3.2: An EMMSAC algorithm is said to be standard if the following holds: $K: \mathcal{P} \rightarrow \mathcal{C}$ is a given controller design procedure satisfying Assumption 2.5(1),(2), $\Delta$ is a given transition delay function and the delay operator $D$ is given by equation (2.16)-(2.19), $l: \mathcal{P} \rightarrow[0,1)$ is a given attenuation function and $K, \Delta, l$ satisfy inequality (2.20) for $1 \leq r<\infty$ and (2.20) for $r=\infty, E$ satisfies Assumptions 2.2(1)-(5) where the interval length of consistency we require from each estimator be given by $\lambda=\max _{p \in U}(2 \Delta(p)+\sigma)$. The switching operator $S=D M(N E, G)$ is given by equations (2.9)-(2.12),(2.13)-(2.15),(2.16)-(2.19). $C$ is defined by equations (2.22),(2.23).

We now come to an intermediate result establishing bounds on the closed loop gain.

Theorem 3.3: Let $1 \leq r \leq \infty$. Suppose $U \subset \mathcal{P}$ and suppose $(H, \nu)$ defines a cover for $U$. Suppose the EMMSAC algorithm is standard. Let $G$ be a plant generating operator that satisfies $p_{*} \in G(j) \subset U, j \leq k$. Suppose $\left(w_{0}, w_{1}, w_{2}\right) \in \mathcal{W} \times \mathcal{W}_{e} \times \mathcal{W}_{e}$ satisfy the closed loop equations (2.1). If

$$
\|c\|\left(\gamma_{4}+\gamma_{5}\right) \chi_{\nu}(H, \nu)<1
$$

and $\alpha_{O P}<1$ then:

$$
\left\|w_{2}\right\| \leq \gamma\left(p_{*}\right)\left\|w_{0}\right\|
$$

where

$$
\begin{aligned}
& \sigma=\sup _{p_{1}, p_{2} \in U} \max \left\{\sigma\left(p_{1}\right), \sigma\left(K\left(p_{2}\right)\right)\right\} \\
& \xi=\left\{\begin{array}{ccc}
r & \text { if } & 1 \leq r<\infty \\
1 & \text { if } & r=\infty
\end{array}\right. \\
& \chi_{\nu}(H, \nu)=2 \sup _{p \in H} \nu(p) \\
& \gamma_{1}\left(p, p_{*}\right)=1+\sup _{\Delta(p) \leq x \leq 2 \Delta(p)} \alpha\left(p_{*}, K(p), 0, x\right) \\
& \gamma_{2}\left(p, p_{*}\right)=\sup _{\Delta(p) \leq x \leq 2 \Delta(p)} \beta\left(p_{*}, K(p), 0, x\right) \\
& \alpha_{O P}=\sup _{p \in U} l(p) \\
& \beta_{O P}=J(\xi) \sup _{p \in U} \sup _{\Delta(p) \leq x \leq 2 \Delta(p)} \beta^{\xi}(p, K(p), x-\sigma, \sigma) \\
& \alpha_{O S}=J(\xi) \sup _{p \in U \Delta(p) \leq x \leq 2 \Delta(p)} \sup _{p(p, K(p), 0, x-\sigma)} \alpha^{\xi}(p)
\end{aligned}
$$

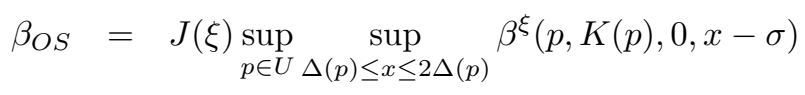

$$
\begin{aligned}
& \gamma_{3}=\left\{\begin{array}{c}
\left(\frac{\left(1+\alpha_{O S}^{1 / r}\right)^{r} \alpha_{O P}}{1-\alpha_{O P}}\right)^{1 / r}+\alpha_{O S}^{1 / r} \\
\text { if } 1 \leq r<\infty \\
\max \left\{1, \alpha_{O S}\right\} \alpha_{O P}+\alpha_{O S} \\
\text { if } r=\infty
\end{array}\right. \\
& \gamma_{4}=\left\{\begin{array}{llc}
\left(\frac{\left(1+\alpha_{O S}^{1 / r}\right)^{r} \beta_{O P}}{1-\alpha_{O P}}\right)^{1 / r} & \text { if } & 1 \leq r<\infty \\
\max \left\{1, \alpha_{O S}\right\} \frac{\beta_{O P}}{1-\alpha_{O P}} & \text { if } & r=\infty
\end{array}\right. \\
& \gamma_{5}=\left\{\begin{array}{ccc}
\beta_{O S}^{1 / r} & \text { if } & 1 \leq r<\infty \\
\beta_{O S} & \text { if } & r=\infty
\end{array}\right. \\
& \gamma_{6}=\frac{1+\gamma_{3}}{1-\|c\|\left(\gamma_{4}+\gamma_{5}\right) \chi_{\nu}(H, \nu)} \\
& \gamma_{7}=\frac{2^{1 / r} \mu|H|^{1 / r}\left(\gamma_{4}+\gamma_{5}\right)}{1-\|c\|\left(\gamma_{4}+\gamma_{5}\right) \chi_{\nu}(H, \nu)} \\
& \gamma\left(p_{*}\right)=\gamma_{6}^{|H|} \prod_{p \in H} \gamma_{1}\left(p, p_{*}\right)\left(|H| \gamma_{7}+\sum_{p \in H} \gamma_{2}\left(p, p_{*}\right)\right)
\end{aligned}
$$

Proof: The (lengthy) proof is omitted, see [2].

The key feature here is that the number of elements in $H$ can be finite (we establish sufficient conditions for this in Section 4), whereas the number of elements in $U$ may be large or infinite (in the case of a continuum), so if $U=H=G$ as in [4], then the bound scales with the number of elements in $G$ which many cases is conservative (for example because of the formal term $|H|$ in the expression for $\gamma$ above).

\section{EXISTENCE OF A FINITE COVER $(H, \nu)$ AND MAIN RESULT}

In order for Theorem 3.3 to establish a finite gain $\gamma$, it is necessary a) for $(H, \nu)$ to be a finite cover, b) the constraint on the size of the neighbourhoods $\nu$ in the cover $(H, \nu)$ specified by the inequality (3.25) to be satisfied and c) the gains $\alpha_{O P}, \beta_{O P}, \alpha_{O S}, \beta_{O S}$ to be finite. In this section we provide sufficient conditions for a), b), c), and constructions for global gain stability and gain function stability.

Definition 4.1: Let $\sigma \in \mathbb{N}$. A control design $K: \mathcal{P} \rightarrow \mathcal{C}$ is said to be $U$ regular if for all $\Delta(p) \leq x \leq 2 \Delta(p)$, the 
functions $l(p), \beta\left(p_{1}, K(p), x-\sigma, \sigma\right), \alpha\left(p_{1}, K(p), 0, x-\sigma\right)$, $\beta\left(p_{1}, K(p), 0, x-\sigma\right), x \in \mathbb{N}$ are continuous with respect to all $p_{1}, p \in U \subset \mathcal{P}$.

Proposition 4.2: Let $U \subset \mathcal{P}$ be compact and suppose $K$ is $U$ regular. Suppose $\left.\chi\right|_{U}$ is continuous. Then there exists a finite cover $(H, \nu)$ of $U$ which satisfies inequality (3.25). Proof Since $U$ is compact and $K$ is $U$ regular, the suprema

$$
\alpha_{O P}=\sup _{p_{1} \in U} l\left(p_{1}\right)
$$

exists and $\alpha_{O P}<1$. Also $\alpha_{O S}, \beta_{O P}<\infty$. Therefore there exist $\epsilon>0$ such that

$$
\epsilon<\left(2\|c\|\left(\gamma_{4}+\gamma_{5}\right)\right)^{-1}
$$

Since $\left.\chi\right|_{U}$ is continuous, $B_{\chi}(p, \epsilon)$ is open and hence $\left\{B_{\chi}(p, \epsilon)\right\}_{p \in U}$ is an open cover of $U$ with respect to the subspace topology of $U$. Since $U$ is compact, there exists a finite set $H \subset U$ such that $\left\{B_{\chi}(p, \epsilon)\right\}_{p \in H}$ covers $U$.

Let $\nu(p)=\epsilon, \forall p \in \mathcal{P}$ hence $(H, \nu) \in\left(\mathcal{P}^{*}, \operatorname{map}\left(\mathcal{P}, \mathbb{R}^{+}\right)\right)$ is a finite cover of $U$. It follows that $\epsilon=\frac{1}{2} \chi_{\nu}(H, \nu)$. Hence inequality (3.25) is satisfied as required.

We now come to our main result:

Theorem 4.3: Let $U \subset \mathcal{P}$ be compact. Suppose the controller design procedure $K: \mathcal{P} \rightarrow \mathcal{C}$ is $U$-regular. Assume the EMMSAC algorithm is standard where $(H, \nu)$ is a cover for $U$ which satisfies inequality (3.25). Let $\gamma$ be as in Theorem 3.3. Then there exists a constant plant generating operator $G$ satisfying $G \subset U$ and $\bar{\gamma} d<1$, where

$$
\begin{aligned}
& \bar{\gamma}=\sup _{p \in U} \gamma(p), \\
& d=\sup _{p_{2} \in U} \inf _{p_{1} \in G} \vec{\delta}\left(p_{1}, p_{2}\right)
\end{aligned}
$$

where the standard EMMSAC design based on $K$ and $G$ stabilises all $P=P_{p_{*}}, p_{*} \in U$ and

$$
\left\|w_{2}\right\| \leq \gamma \frac{1+d}{1-\bar{\gamma} d}\left\|w_{0}\right\|
$$

Proof: Since $U$ is compact there exists a constant, finite plant generating operator $G$ such that $G \subset U, k \in \mathbb{N}$ and such that $\bar{\gamma} d<1$. Let $p_{1} \in G$ be such that $\vec{\delta}\left(p_{*}, p_{1}\right)<d$. Since $G \subset U, k \in \mathbb{N}$ it follows by Theorem 3.3 that

$$
\left\|\Pi_{P_{p_{1}} / / C}\right\| \leq \gamma\left(p_{1}\right) \leq \bar{\gamma}<\infty .
$$

Since $\vec{\delta}\left(p_{*}, p_{1}\right)<d<\bar{\gamma}^{-1}=b_{P, C}$ the result follows from Theorem 2.1 as required.

We again return to the example plant given by equation (3.24) with $b=1$ and $a \in\left[-a_{\max }, a_{\max }\right]=U$. by Proposition 4.2 there exists a finite, constant cover $(H, \nu)$. We could let $G=U$ and achieve a finite gain bound, however since $U$ is a continuum the EMMSAC controller is infinite dimensional hence not implementable. However by Theorem 4.3 there exists a finite plant model set $G \subset U$ such that a finite gain bound can be achieved.
Although we have stated this theorem as an existence result, the proof is constructive, and can be used to show that a common gain bound exists for $G=R_{l}$, where $l>l^{*}$ for some suitable threshold $l^{*}>0$. This implies that $G$ can be refined off-line where the achieved gain bound is invariant to the refinement level (e.g. $l>l^{*}$ ) since it depends on the complexity of $U$ as measured by the cover $(H, \nu)$. The construction utilised in the proof also leads to the beginnings of a principled designed methodology, see [2].

\section{CONCLUSION}

Key challenges (see e.g. [1], [5], [2]) in multiple model adaptive control are 1 . the construction of comprehensive stability, robustness and performance results, and 2 . the performance orientated design of candidate plant model sets. Both issues are closely related since the lack of a suitable bounds of global performance precludes fully principled performance orientated design. The ideas behind using covers of uncertainty sets as the basis for the design can also be found in e.g. [1], [5]: note that in contrast to these contributions, we establish global performance results based on these covers, hence leading to the promise of a fully principled design methodology [2].

\section{REFERENCES}

[1] B. D. O. Anderson, T. S. Brinsmead, F. De Bruyne, J. P. Hespanha, D. Liberzon, and A. S. Morse. Multiple model adaptive control. part 1: Finite controller coverings. Int. J. of Robust and Nonlinear Control, 10:909-929, 2000.

[2] D. Buchstaller. Robust Stability and Performance for Multiple Model Switched Adaptive Control. PhD thesis, University of Southampton, School of Electronics and Computer Science (ECS), 2009. Submitted.

[3] D. Buchstaller and M. French. Scaling of gain bounds for switched adaptive control with large uncertainties. In Proc. of the 46th IEEE Conf. on Decision and Control (CDC) in New Orleans, LA, USA, pages 915-920, 2007.

[4] D. Buchstaller and M. French. Gain bounds for multiple model switched adaptive control of general MIMO LTI systems. In Proc. of the 47th IEEE Conf. on Decision and Control (CDC) in Cancun, Mexico, pages 5330-5335, 2008.

[5] S. Fekri, M. Athans, and A. Pascoal. Issues, progress and new results in robust adaptive control. Int. J. of Adaptive Contr. and Signal Processing, 20(10):519-579, 2006.

[6] T. P. Fisher-Jeffes. Multiple-Model Switching Control to Achieve Asymptotic Robust Performance. PhD thesis, University of Cambridge, 2003.

[7] M. French and S. Trenn. $l^{p}$ gain bounds for switched adaptive controllers. In Proc. of the 44th IEEE Conf. on Decision and Control $(C D C)$ and the European Control Conf. (ECC) in Seville, Spain, pages 2865-2870, 2005.

[8] T. T. Georgiou and M. C. Smith. Robustness analysis of nonlinear feedback systems: An input-output approach. IEEE Transactions on Automatic Control, 42(9):1200-1220, September 1997.

[9] J. P. Hespanha, D. Liberzon, and A. S. Morse. Overcoming the limitations of adaptive control by means of logic-based switching. System and Control Letters, 49(1):49-65, 2003.

[10] D. Liberzon. Switching in Systems and Control. Birkhauser, 2003.

[11] A. S. Morse. Supervisory control of families of linear set-point controllers - part 1: Exact matching. IEEE Transactions on Automatic Control, 41(10):1413-1431, October 1996.

[12] A. S. Morse. Supervisory control of families of linear set-point controllers - part 2: Robustness. IEEE Transactions on Automatic Control, 42(11):1500-1515, November 1997.

[13] G. Vinnicombe. Examples and counterexamples in finite $\mathcal{L}_{2}$-gain adaptive control. Leuven: Sixteenth International Symposium on Mathematical Theory of Networks and Systems (MTNS2004), 2004. 